\title{
Simulation Modeling for Optimal Control of Additive Manufacturing Processes
}

\author{
Jinkun Lee ${ }^{\mathrm{a}, *}$, Felipe Lopez ${ }^{\mathrm{b}}$, Peter Denno ${ }^{\mathrm{b}}$, Vittaldas Prabhu ${ }^{\mathrm{a}}$ \\ ${ }^{a}$ Industrial and Manufacturing Engineering, The Pennsylvania State University, PA 16802 \\ ${ }^{b}$ System Integration Division, National Institute of Standards and Technology, MD 20899
}

\begin{abstract}
Simulation models of direct energy deposition and powder bed fusion processes have been developed to investigate dynamic control at the process level. A meta-model, developed from computational experiments with an existing numerical model, is used to characterize the physics at the hot spot beneath a laser beam. The meta-model describes model the process dynamics at the millisecond scale using closed-form analytic equations to which we add un-modeled dynamics in physical processes. An auxiliary finite difference model of heat transfer simulates heating of regions adjacent to the laser track. Temperatures predicted with the auxiliary model are incorporated as initial conditions for meta-model predictions of future laser scans. The synergy of the metamodel and the finite difference model creates a higher-fidelity model, which is used to generate input data for a model-free optimal controller. Simulation results prove the capability of proposed optimal controller to vary scan speed accordingly when accounting for track-to-track interaction.
\end{abstract}

Keywords: additive manufacturing, simulation model, optimal control, laser powder bed fusion

\section{Introduction}

Advanced manufacturing has rapidly become an active research area in the United States since the President's Council of Advisors on Science and Technology (PCAST) report in June 2011 [1]. Additive Manufacturing (AM) has attracted attention due to its advantages in cost reduction, adaptive design and development, and diversity in product design, as compared to traditional manufacturing. Still, to achieve global competitiveness, AM needs to meet additional demands from contemporary issues such as energy and process level intelligence. Handling such issues requires advanced process control from the integration of high performance computing for modeling, simulation, and analysis. In particular, optimal control may address the energy issue by searching for the most energy efficient process control trajectory. More importantly, if a model-free control technique automatically finds an optimal process from sensorcollected data without a priori process dynamic models, the manufacturing process could be regarded as having an intelligence to self-adjust for optimality.

In this paper, a recently developed single input single output (SISO) model-free optimal control technique is utilized [2]. The merit of this technique is its capability to find optimal solution of a black-box dynamic system such as AM process with only observation data. That means, this model-free technique is expected to work with any type of numerical model as long as it produces an observational data set. The ultimate goal of this technique is to obtain automatic optimal control trajectories of AM processes through iterative optimization without needing to identify the explicit dynamics. Technically, this model-free

\footnotetext{
${ }^{*}$ Corresponding author

Email address: jinkunlee@psu.edu (Jinkun Lee)
}

approach needs only time series observation data sequences of control and state variables.

The selection of control and state variables is determined by their proper relationship as an input and an output of the system. In many AM processes, it is still not clear how much influence each factor has on the build quality of AM product. For example, $\mathrm{Hu}$ [3] set laser power as a control variable for feedback control while other parameter values such as scan speed and powder feed rate are fixed in order to maintain a uniform melt-pool size, which lead to a uniform build shape. Simch [4] studied a relation between build density and laser energy input power which includes several control variables such as laser power, scan rate, scan line spacing, and layer thickness.

A recent NIST report [5] shows correlation between control variables and product qualities through a number of process signature parameters. Some investigations have given hints to identify the convoluted relations between them. Gong [6] tried to show the relationships between beam speed, temperature profile, and microscopic build quality through experimental data of four different beam speeds. Even though the explicit relationship was not modeled quantitatively, the result showed that two specific beam speeds with similar temperature profile peak values resulted in best quality. This implies that the build quality may be achieved by controlling the peak value of temperature profile, which is again controlled by beam speed. Therefore, a laser scan speed and peak temperature beneath the laser beam have been selected as control and state variables respectively in this study.

The observational data for the development of process models is obtained from either numerical process simulation or real experiment. Recent empirical research has been reporting both the opportunity and challenge of a thermographic measurement 
of surface temperature in AM processes. Price [7] applied a near infrared (NIR) thermography to an electron beam additive manufacturing (EBAM) process to measure the temperature profile across the melt-pool for assumed emissivity of Ti$6 \mathrm{Al}-4 \mathrm{~V}$ powder and the NIR transmission ratio of the glasses. Lane [8] suggested the use of hyperspectral camera as well as traditional IR camera to determine temperature and emissivity by comparing intensity versus wavelength plot with Planck's radiation law. Rodriguez [9] put further efforts into the use of in-situ infrared thermography for a specific electron beam melting system by developing a case-dependent thermal model that calculates mean radiant temperature, which is then used to correct IR parameters to determine powder emissivity. Until now, uncertainty in the determination of emissivity compromises the accuracy of thermographic measurements, limiting their applicability [8].

In lieu of observational data, we use data generated from a simulation model for the model-free control technique until the experimental data from thermographic measurement is readily available. A control path observation, a pair of control sequence and resulting state sequence, are generated by using numerical simulation models. The main advantage of numerical simulation is the time and cost saving in the model-free optimal control since this technique requires iterative process data until the control path converges to an optimal trajectory. Simulation models of direct energy deposition (DED) and direct metal laser sintering (DMLS) processes have been developed to investigate a dynamic control at the process level with various objectives such as constant processing temperature, minimum energy use, or minimum laser speed change.

\section{Simulation model}

\subsection{Process meta-model for laser scan temperature}

For a DED process, a laser-based free-forming process with Titanium powder is considered. Koomsap [10] proposed a process meta-model using a polynomial regression with 27 data points to run a model-based simulation with reduced computational cost. The reported error of the proposed meta-model is within $3 \%$. The data points have been obtained from a finite difference method (FDM) code developed by Semak et. al.[11], which simulates the motion of the melt-pool and temperature distribution. Several main assumptions of the FDM code includes: different temperature independent material properties in solid and liquid phases, laser power absorption on the surface (no volumetric heat generation), and melt layer as a thin boundary layer. The simulation results have been verified with experimental observations and data.

The transient meta-model (1) computes transient peak temperature beneath the laser beam $T$ as a function of time. The steady state temperature $T_{s}$ and the time constant $\tau$ in the transient meta-model (1) are determined from regression formulas (2) and (3) with process parameters such as laser power $P(k W)$, translation speed $V(\mathrm{~cm} / \mathrm{s})$, and powder flow rate $M(\mathrm{~g} / \mathrm{s})$. The meta-model (1) approximates the transient time delay in surface temperature by using the time constant $(\tau)$, and it approaches the steady-state temperature $\left(T_{s}\right)$ as time $(t)$ increases.

$$
T(t)=f(P, V, M, t)=T_{s}\left(1-e^{-t / \tau}\right)
$$

$$
T_{s}=\mathbf{H}_{\mathbf{s}}{ }^{T} \mathbf{X}_{\mathbf{s}}+2608.7
$$

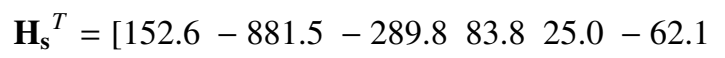$$
-7.6-1.048 .2-2.6-5.412 .1]
$$$$
\mathbf{X}_{\mathbf{s}}{ }^{T}=\left[P V M P V P M M V P^{2} P^{2} V V^{2} P M V P V^{2} M V^{2}\right]
$$

$$
\begin{aligned}
& \tau=\mathbf{h}_{\mathbf{t}}^{T} \mathbf{x}_{\mathbf{t}}+0.2239 \\
& \mathbf{h}_{\mathbf{t}}{ }^{T}=\left[\begin{array}{llll}
-0.0249-0.0696-0.0091 & 0.0086 & 0.0007
\end{array}\right.
\end{aligned}
$$

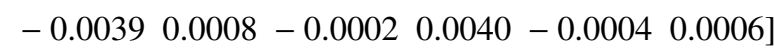

$$
\begin{aligned}
& \mathbf{x}_{\mathbf{t}}{ }^{T}=\left[P V M P V P M M V P^{2} P^{2} V V^{2} P V^{2} M V\right]
\end{aligned}
$$

However, the original model equation lacks the capability of treating variable initial conditions since it always starts from $0 \mathrm{~K}$ when $t=0$. In order to incorporate the initial temperature effect, the transient model has been modified by shifting the transient time horizon with a time constant $\left(t_{0}\right)$, the time when the initial temperature is met in the original meta-model. The modified transient meta-model (4) with $t_{0}$ now begins with the specific initial condition $T_{0}$ at $t=0$, while following the original meta-model for the rest of simulation. Since $T_{s}$ and $\tau$ in (2) and (3) have not been changed, the error of this modified model remains unchanged.

Fig.1 shows the steady state and transient surface temperature changes in a DED process with an oscillating laser scan speed as control input while the other parameters are fixed $(P=7 \mathrm{~kW}, M=0.1 \mathrm{~g} / \mathrm{s})$. The reason for selecting a sinusoidal control input is to check whether the meta-model returns reasonable results from a thermodynamic point of view. The modified model shows a transient temperature increase from the given initial temperature while converging to the steady state temperature. The sinusoidal control sequence shows rapid temperature increase when the laser scan speed is low and vice versa. The result looks reasonable as heat absorption rate is inversely proportional to the translation speed of heat source.

$$
\begin{aligned}
& T(t)=T_{s}\left(1-e^{-\left(t_{0}+t\right) / \tau}\right) \\
& t_{0}=-\tau \ln \left(1-T_{0} / T_{s}\right)
\end{aligned}
$$

\subsection{Heat transfer model for temperature profile}

In powder bed fusion (PBF) processes, a similar meta-model can be used with appropriate regression parameters. In this work, DMLS has been selected as the case of several PBF processes. DMLS is distinct from DED in that it is conducted in a bed of powdered metal often with a parallel scanning pattern which makes scan line spacing another energy input parameter [4]. This difference makes it necessary to consider heat transfer phenomena in the adjacent or previously scanned area because the remaining or transferred heat in such area may result in a different initial temperature profile for the next laser scan. 


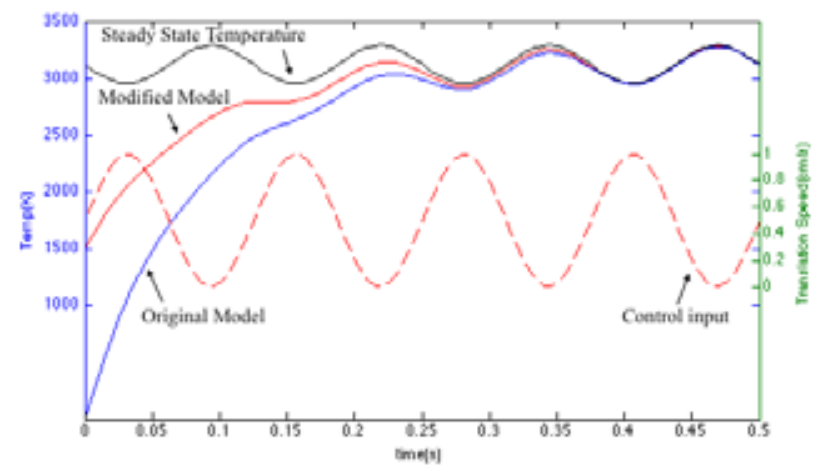

Figure 1: Transient process model

The governing heat equation is shown in (5), where $\rho, C_{p}, k$, and $q$ denote density, heat capacity, thermal conductivity, and absorbed heat flux, respectively.

$$
\frac{\partial T}{\partial t}=\frac{k}{c_{p} \rho} \nabla^{2} T+\frac{1}{c_{p} \rho} q=\alpha\left[\nabla^{2} T+\frac{q}{k}\right]
$$

The heat equation (5) is solved numerically by using a finite difference method (FDM). A computationally inexpensive forward-time central-space (FTCS) scheme has been selected to reduce simulation time within affordable accuracy. In (6), the superscript and subscript of temperature variable $T$ denote time step and space position respectively. The stability condition $\max \left(\alpha \frac{\Delta t}{\Delta x^{2}}, \alpha \frac{\Delta t}{\Delta y^{2}}, \alpha \frac{\Delta t}{\Delta z^{2}}\right) \leq \frac{1}{2}$ is satisfied by adjusting time and space mesh size. In the heat transfer treatment on the boundary, identical heat flux with adjacent nodes is applied to simulate continuous heat transfer toward exterior region of current mesh.

$$
T_{i, j, k}^{n+1}=T_{i, j, k}^{n}+\alpha \Delta t\left[\frac{\delta^{2} T_{i}^{n}}{\Delta x^{2}}+\frac{\delta^{2} T_{j}^{n}}{\Delta y^{2}}+\frac{\delta^{2} T_{k}^{n}}{\Delta z^{2}}+\frac{q_{i, j, k}^{n}}{k}\right]
$$

(where $\delta^{2} f_{i}=f_{i-1}-2 f_{i}+f_{i+1}$ )

The FDM model (6) has been compared with a finite element model (FEM) developed by Shen and Chou [12] for $T i-6 A l-4 V$. Shen and Chou applied temperature dependent thermal properties into their model in order to incorporate the latent heat of fusion. In our FDM model, temperature independent fixed parameters are used for two-dimensional (2D) and three-dimensional (3D) simulations to reduce the complexity of model and computation time because our main concern is to obtain the temperature profile after a laser beam scan for following process control. The 3D simulation result is for a layer thickness of $2 \mathrm{~mm}$ with Neumann boundary conditions. The parameters used in our FDM model are listed in Table 1. The listed values are within the range of temperature dependent property values of Shen and Chou's model. Fig.2 shows that simulation results agree well in the vicinity of the laser beam center while there is some discrepancy in the tail. This might be due to the use of fixed parameter values in our model. It is worth to mention that the simulation result of Shen and Chou is for a layer thickness of $0.1 \mathrm{~mm}$. Therefore, the result of our 2D model fits better than that of 3D result. The higher temperature
Table 1: Parameters used in the FDM model

\begin{tabular}{lr}
\hline Parameters & Values \\
\hline Density $\left(\mathrm{kg} / \mathrm{m}^{3}\right)$ & 4506 \\
Specific Heat $(\mathrm{J} / \mathrm{kgK})$ & 750 \\
Conductivity $(\mathrm{W} / \mathrm{mK})$ & 30 \\
Scan speed $(\mathrm{mm} / \mathrm{s})$ & 400 \\
Preheat temperature $(K)$ & 1033 \\
\hline
\end{tabular}

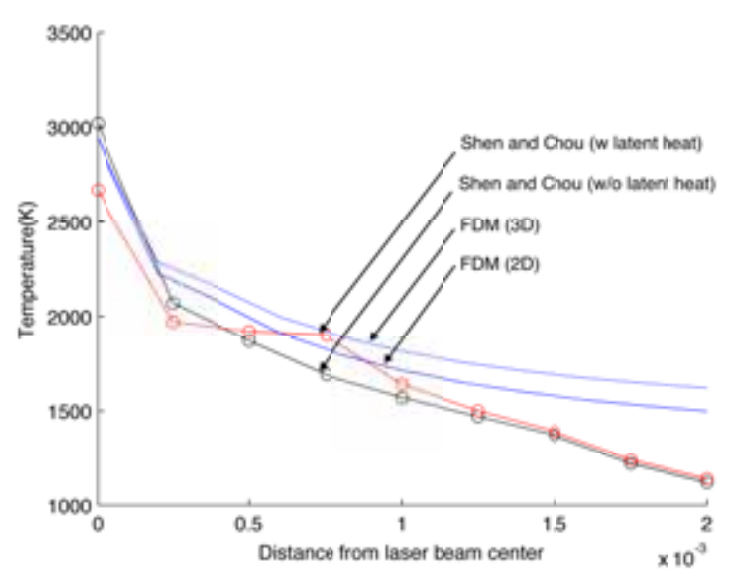

Figure 2: Temperature profile from laser beam center

profile of 3D result can be explained by a reduced temperature gradient in the vertical direction as shown in Fig.3. The 2D model will be useful when simulating a track-to-track scan in a thin layer while 3D model simulating a layer by layer stacking.

Since the power of laser beam is a fixed parameter in many AM processes while the laser scan speed may vary, the value of heat flux $q$ in (6) is determined from the nominal laser power, absorption efficiency, and heat absorbing area. Simulation results from the FDM model show that the amount of heat absorption is proportional to the amount of duration time. Regarding laser scanning hatch line directions, unidirectional and bidirectional cases are considered. In the simulation, the laser scans from left to right direction repeatedly in the unidirectional case while it changes directions alternately in the bidirectional case.

Fig. 4 shows the temperature beneath the laser beam after several laser scan processes in 2D FDM model. Same parameters values in Table 1 are used in this simulation. The numbers and directions of the processes are illustrated with arrows at the top of each graph. The red colored peak on the surface plot is the laser beam scanning position with an exponentially decreasing temperature profile behind. The material temperature ahead of laser beam is higher than preheat temperature due to remaining heat from previous scan. The temperature on the boundaries can be also higher than the preheat temperature since it is assumed that the heat transferring area is not limited by the numerical mesh size by applying Neumann boundary condition. The main reason of considering a heat transfer model is to estimate surface temperature profile from previous laser scan so that it may be fed back to a new scan as an initial condition. A specific build scenario of track to track laser scanning has been investigated to see the effect of different scan directions. Fig.5 


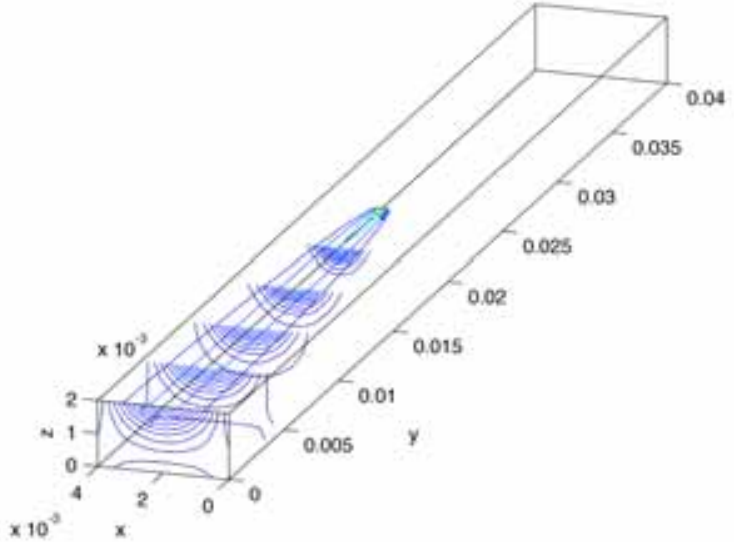

Figure 3: Isothermal contour map in 3D model

shows the traces of peak temperature along laser scan path. (a) Unidirectional laser scan shows that the peak temperature keeps increasing uniformly due to the elevation of initial temperature profile from the preceding scan. The increasing rate of peak temperature reduces toward an equilibrium state as the iteration number increases. (b) Bidirectional laser scan shows that the peak temperatures on both ends show steep gradients because the laser beam reheats those areas right after turning its direction. As a result, the initial temperature profile in front of the current laser scan direction shows an exponential decrease. From a control view point, this varying peak temperature due to the temperature profile of preceding scan plays an important role in controlling the subsequent laser scan speed for the build quality in boundary.

\section{Optimal Process Control}

\subsection{Model-Free Optimal Control Algorithm}

A SISO model-free optimal control approach proposed by Lee [2] has been applied to the aforementioned laser based, free-forming process model. The laser scan speed is often selected as one of the control variables for quality in AM applications [13][14][15]. The AM process is formulated as an optimal control problem (7) with laser scan speed $V(\mathrm{~cm} / \mathrm{s})$ as a control variable $u$ and surface temperature $T(K)$ as a state variable $x$. The proposed approach is a local search algorithm which finds an improving control trajectory in the vicinity of current control trajectory. Therefore, it solves a constrained optimization problem even if the control variable set $U$ is not explicitly declared. In the problem (7), $t_{0}$ and $t_{f}$ are initial and terminal times respectively, $x_{0}$ is an initial state condition at $t_{0}, \phi\left(x\left(t_{f}\right), t_{f}\right)$ is a penalty function for terminal state, and $L(x(t), u(t), t)$ is the Lagrangian which is an objective functional integrand. It is assumed that all the variables are continuous and twice-differentiable functions with respect to time, reachability condition is satisfied for every initial value, and therefore there

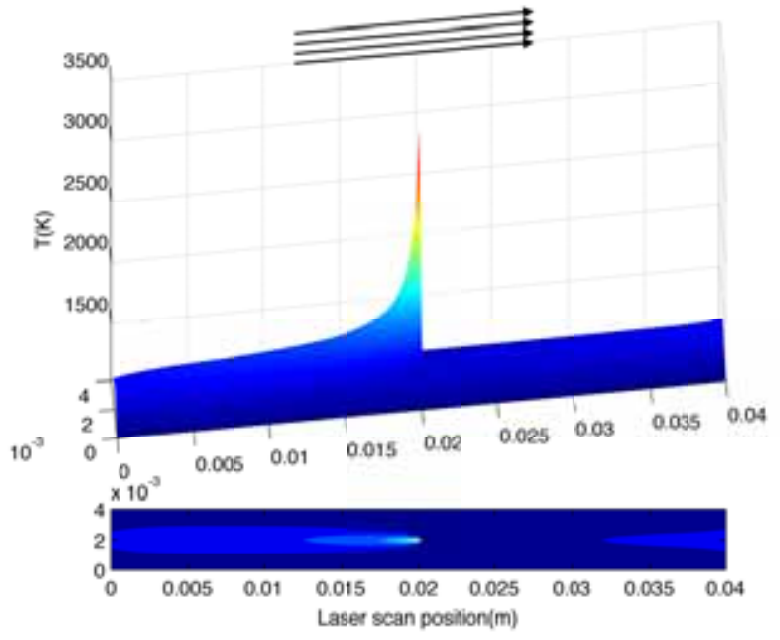

(a) Unidirectional laser scan

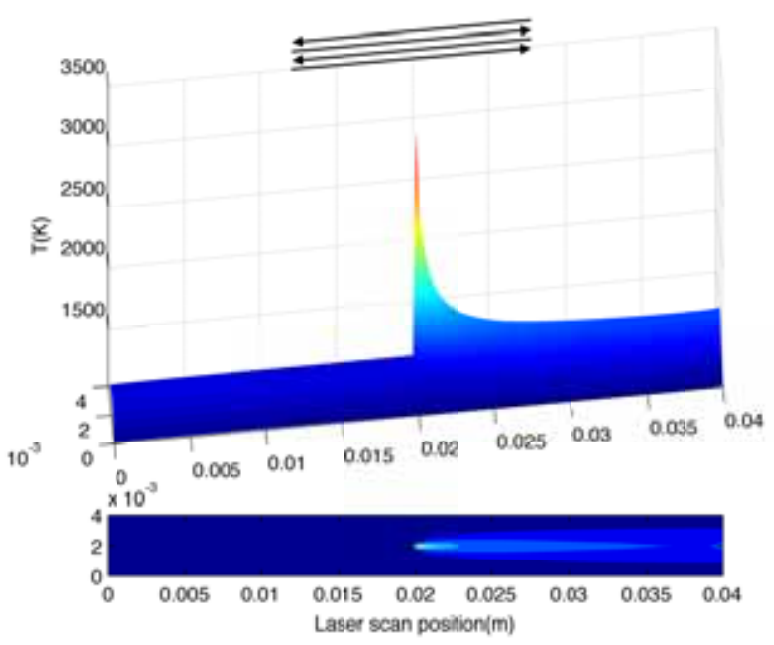

(b) Bidirectional laser scan

Figure 4: Heat transfer simulation for different laser scan directions

exists a unique solution.

$$
\begin{array}{ll}
\min _{u} & J=\phi\left(x\left(t_{f}\right), t_{f}\right)+\int_{t_{0}}^{t_{f}} L(x(t), u(t), t) d t \\
\text { s.t. } & \dot{x}(t)=f(x(t), u(t), t) \\
& x\left(t_{0}\right)=x_{0}, \quad x \in X, u \in U
\end{array}
$$

The model-free techniques use only control and state data sequence which is from the aforementioned simulation model. All variables and functions in this algorithm are treated as sequences with an identical time interval between data points. In order to estimate $f(x(t), u(t), t)$, a central difference scheme is applied to the state sequence $x(t)$, that is, temperature change with respect to time. The estimated sequence of $f$ is denoted as $\bar{f}$. The state and control variables, $x(t)$ and $u(t)$, are functions with respect to time, and they are often used as arguments of a functional. For convenience, $x(t)$ and $u(t)$ will be denoted sim- 


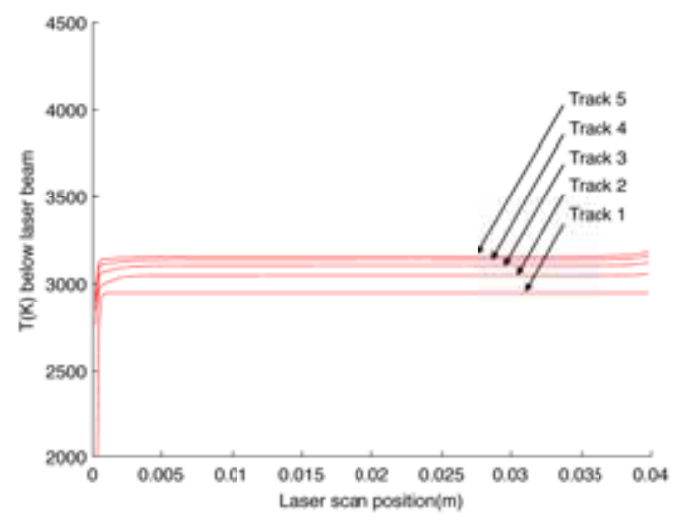

(a) Unidirectional laser scan

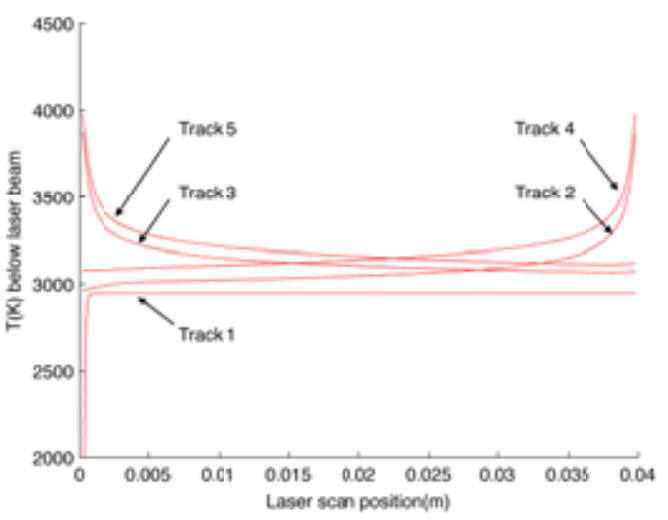

(b) Bidirectional laser scan

Figure 5: Simulation results of peak temperature

ply by $x$ and $u$ when they are arguments of a functional such as $f(x, u, t)$ or $L(x, u, t)$.

$$
\begin{aligned}
& \bar{f}(x, u, t)=\dot{x} \\
& \bar{f}^{n}=\frac{\delta x^{n}}{2 \Delta t} \\
& \left(\text { where } \delta x^{n}=x^{n+1}-x^{n-1}\right)
\end{aligned}
$$

Another control path $(u(t), \hat{x}(t))$ is generated from a perturbed initial condition without changing current control sequence. The perturbed state sequence is denoted by a hat over a letter. The partial derivative estimate $\partial \bar{f}(x, u, t) / \partial x(t)$ is obtained from a pair of original and perturbed paths by (9) per iteration.

$$
\frac{\partial \bar{f}(x, u, t)}{\partial x}=\frac{\bar{f}(\hat{x}, u, t)-\bar{f}(x, u, t)}{\hat{x}-x}
$$

(where $u$ : fixed control sequence per iteration)

The adjoint $\lambda(t)$ is then estimated by (10) with $L(x, u, t)$ which is the objective functional integrand. Since $L(x, u, t)$ is given as an analytic function, its derivative sequence $\partial L / \partial x$ is analytically found by plugging in $x(t)$ and $u(t)$ into the derivative. The estimated $\bar{\lambda}(t)$ is numerically computed in a backward

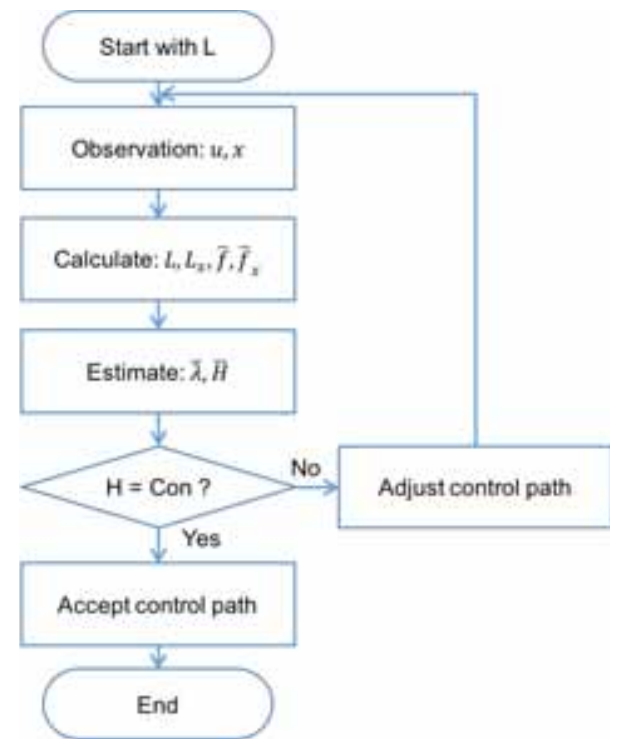

Figure 6: Flow chart of model-free algorithm

direction. The Hamiltonian is finally estimated by (11).

$$
\begin{aligned}
-\dot{\lambda}(t) & =\frac{\partial \bar{f}(x, u, t)}{\partial x} \lambda+\frac{\partial L(x, u, t)}{\partial x} \\
\bar{\lambda}(t-1) & =\left[1+\Delta t \frac{\partial \bar{f}}{\partial x}(t)\right] \bar{\lambda}(t)+\Delta t \frac{\partial L}{\partial x}(t)
\end{aligned}
$$

$$
\bar{H}(x, u, \lambda, t)=\bar{\lambda}(t) \bar{f}(x, u, t)+L(x, u, t)
$$

The constant Hamiltonian, alternative necessary condition of optimality, is tested with the Hamiltonian estimation. If the condition is not met, the control variable $u(t)$ is regulated through iterations until its resulting Hamiltonian estimation becomes constant. Fig. 6 shows the flow chart of the algorithm.

\subsection{Optimal Control with Simulation Data}

In the simulation study, two target temperatures, around and above Titanium powder's melting point (1900 $\mathrm{K}$ and $2200 \mathrm{~K}$ ) have been considered as process conditions. The steady state laser scan speeds $(4.65 \mathrm{~cm} / \mathrm{s}$ and $3.5 \mathrm{~cm} / \mathrm{s})$ for the target temperatures have been found respectively from the steady state meta-model for a specific test condition $(P=7 \mathrm{~kW} / \mathrm{s}$ and $M=0.1 \mathrm{~g} / \mathrm{s})$. Fig. 7 shows the transient times $(0.128 s$ and $0.222 s$ ) to reach corresponding target temperatures resulting from the transient meta-model without laser scan speed control. There exists an opportunity for the optimal control in this transient time period.

Based on this transient laser scan characteristics, two optimization cases are considered as optimal control objectives: (i) minimize the control time to reach the target temperature and (ii) minimize control variations until the target temperature is reached after the same transient time. The objective function for the first case is formulated as (12), and the second case as 


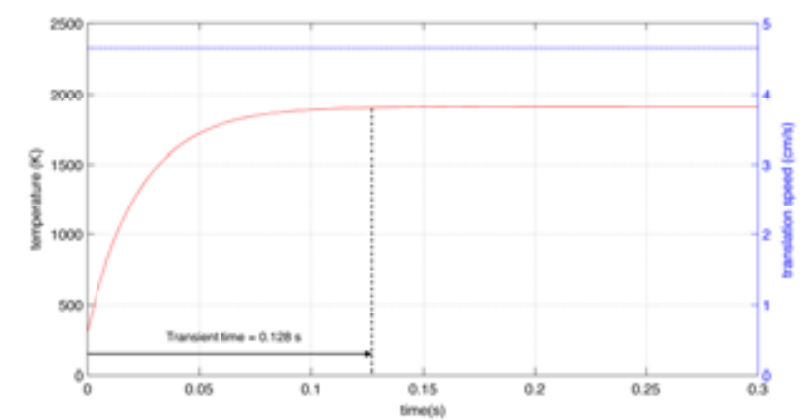

(a) Target temperature $1900 \mathrm{~K}$ (scan speed $4.65 \mathrm{~cm} / \mathrm{s}$ )

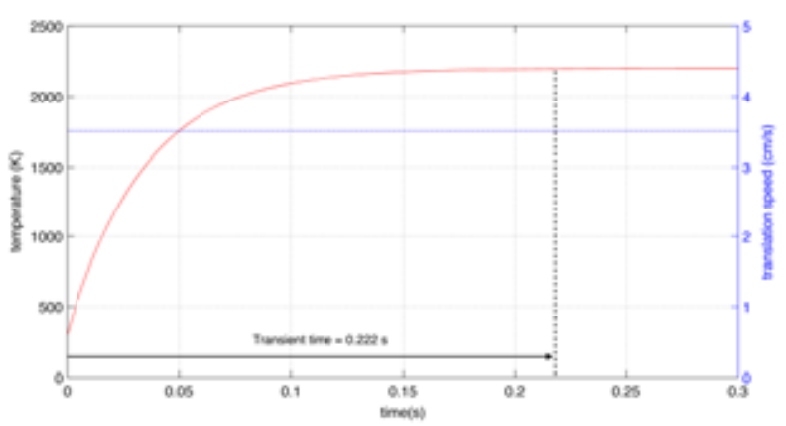

(b) Target temperature $2200 \mathrm{~K}$ (scan speed $3.5 \mathrm{~cm} / \mathrm{s}$ )

Figure 7: Transient time to target temperature

(13).

$$
\begin{aligned}
& J_{1}=\phi\left(x\left(t_{f}\right), t_{f}\right)+\int_{t_{0}}^{t_{0}+t} 1 d t \\
& J_{2}=\phi\left(x\left(t_{f}\right), t_{f}\right)+\int_{t_{0}}^{t_{f}} \frac{1}{2} u(t)^{2} d t
\end{aligned}
$$

The results of model-free optimal control for the minimum time objective are shown from Fig.8 to Fig.11. Fig.8 and Fig.10 show control trajectories on Lagrangian function surface with respect to $(u, x)$ coordinates for the two target temperature cases. The improved control paths reach the target temperatures after $64 \mathrm{~ms}$ while the transient meta-model without control takes $128 \mathrm{~ms}$ for the $1900 \mathrm{~K}$ case; $106 \mathrm{~ms}$ instead of $222 \mathrm{~ms}$ for the $2200 \mathrm{~K}$ case. Initial control trajectories in both cases are feedback control trajectories which have been used as the initial conditions of model-free control technique to find local optimal solutions. Fig.9 and Fig.11 show state and control value changes with respect to control time. Several control trajectories during iteration steps show the search process of model-free technique for a better control trajectory until the last one is found without further improvement. Again, the model-free technique searches an optimal solution based on recent control trajectory data. Therefore, the search result is a local optimal solution. Similarly, Fig.12 to Fig.15 show the results for the minimum control variance objective. The state variables with respect to time are similar with those of the initial control trajectories respectively. However, the control variables are distinctively different from them and show almost constant lines with a very small slope, which means minimum changes of laser scan speed during the processes.

\section{Conclusive Remarks}

Simulation models have been developed to investigate a dynamic control at the process level of laser-based additive manufacturing. An existing transient meta-model developed by Koomsap [10] has been slightly modified to incorporate initial conditions, and a heat transfer FDM model has been developed to consider the effect of preceding temperature profile on following laser beam scan. The inclusion of FDM model helps us to extend our AM process models from direct energy deposition (DED) to power bed fusion (PBF) processes.

The simulation models generate control and state sequence data, and the model-free optimal controller iteratively finds a local optimal control trajectory which improves an objective function. Based on simulation models, minimum time and minimum control variance objectives have been investigated for two different target temperature cases. The final control trajectory after a few iterations shows significant improvement over initial control trajectory in the objective function values. This study is a simulation-based optimization with optimal control. In order to improve the accuracy of simulation model, we plan to incorporate phase changes in melting and re-solidification into our FDM model by using temperature dependent material properties. The refined numerical model might be used to develop another meta-model to reduce the computation time for an almost real-time control.

The model-free controller in this study works for a black-box system where only input and output data is obtainable. Therefore, the proposed process control framework is open to any type of AM process model and real time data measurement. Current study of process optimization is based on the simulation models because of not only the expensive experimental cost but also the difficulty in measuring accurate surface temperature. We plan to further develop this framework for a datadriven optimization in the near future.

\section{Acknowledgment}

This research was supported by National Institute of Standards and Technology.

\section{References}

[1] A. Anderson, Report to the President on Ensuring American Leadership in Advanced Manufacturing, Executive Office of the President, 2011. URL http: //eric.ed.gov/?id=ED529992

[2] J. Lee, J. Singh, V. Prabhu, Automatic optimal control of Field Assisted Sintering Technology, in: 2014 IEEE International Conference on Automation Science and Engineering (CASE), 2014, pp. 764-769. doi:10.1109/CoASE.2014.6899412.

[3] D. Hu, R. Kovacevic, Sensing, modeling and control for laser-based additive manufacturing, International Journal of Machine Tools and Manufacture 43 (1) (2003) 51-60. doi:10.1016/S0890-6955(02)00163-3.

[4] A. Simchi, Direct laser sintering of metal powders: Mechanism, kinetics and microstructural features, Materials Science and Engineering: A 428 (12) (2006) 148-158. doi:10.1016/j.msea.2006.04.117.

[5] M. Mani, B. Lane, A. Donmez, S. Feng, S. Moylan, R. Fesperman, Measurement Science Needs for Real-time Control of Additive Manufacturing Powder Bed Fusion Processes, Tech. Rep. NISTIR 8036 (2015). URL http://dx.doi.org/10.6028/NIST. IR. 8036 
[6] X. Gong, J. Lydon, K. Cooper, K. Chou, Beam speed effects on ti6al4v microstructures in electron beam additive manufacturing, Journal of Materials Research 29 (17) (2014) 1951-1959. doi:10.1557/jmr.2014.125.

[7] S. Price, K. Cooper, K. Chou, Evaluations of temperature measurements by near-infrared thermography in powder-based electron-beam additive manufacturing, in: Proceedings of the Solid Freeform Fabrication Symposium, University of Texas, Austin, TX, 2012, pp. 761-773.

[8] B. Lane, S. Moylan, E. Whitenton, L. Ma, Thermographic measurements of the commercial laser powder bed fusion process at NIST, in: International Solid Freeform Fabrication Symposium, 2015, pp. 575-591.

[9] E. Rodriguez, J. Mireles, C. A. Terrazas, D. Espalin, M. A. Perez, R. B. Wicker, Approximation of absolute surface temperature measurements of powder bed fusion additive manufacturing technology using in situ infrared thermography, Additive Manufacturing 5 (2015) 31-39. doi:10.1016/j.addma.2014.12.001

[10] P. Koomsap, V. V. Prabhu, J. T. Schriempf, E. W. Reutzel, Simulationbased design of laser-based free forming process control, Journal of Laser Applications 13 (2) (2001) 47-59. doi:10.2351/1.1356422.

[11] V. V. Semak, B. Damkroger, S. Kempka, Temporal evolution of the temperature field in the beam interaction zone during laser material processing, Journal of Physics D: Applied Physics 32 (15) (1999) 1819 doi:10.1088/0022-3727/32/15/309.

URL http://stacks.iop.org/0022-3727/32/i=15/a=309

[12] N. Shen, K. Chou, Thermal Modeling of Electron Beam Additive Manufacturing Process: Powder Sintering Effects (2012) 287295doi:10.1115/MSEC2012-7253.

URL http://dx.doi.org/10.1115/MSEC2012-7253

[13] J. Gockel, J. Beuth, K. Taminger, Integrated control of solidification microstructure and melt pool dimensions in electron beam wire feed additive manufacturing of Ti-6al-4v, Additive Manufacturing 14 (2014) 119-126. doi:10.1016/j.addma.2014.09.004

[14] H. Gong, K. Rafi, H. Gu, T. Starr, B. Stucker, Analysis of defect generation in Ti6al4v parts made using powder bed fusion additive manufacturing processes, Additive Manufacturing 14 (2014) 87-98. doi:10.1016/j.addma.2014.08.002

[15] A. Fathi, A. Khajepour, E. Toyserkani, M. Durali, Clad height control in laser solid freeform fabrication using a feedforward PID controller, International Journal of Advanced Manufacturing Technology 35 (3/4) (2007) 280-292. doi:10.1007/s00170-006-0721-1.
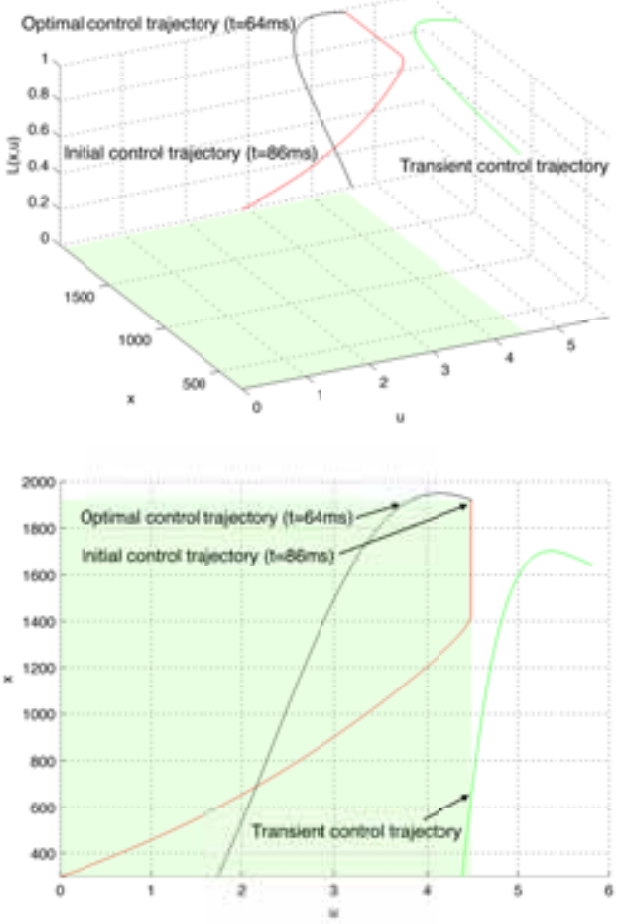

Figure 8: Control trajectory plot (min time for 1900K)
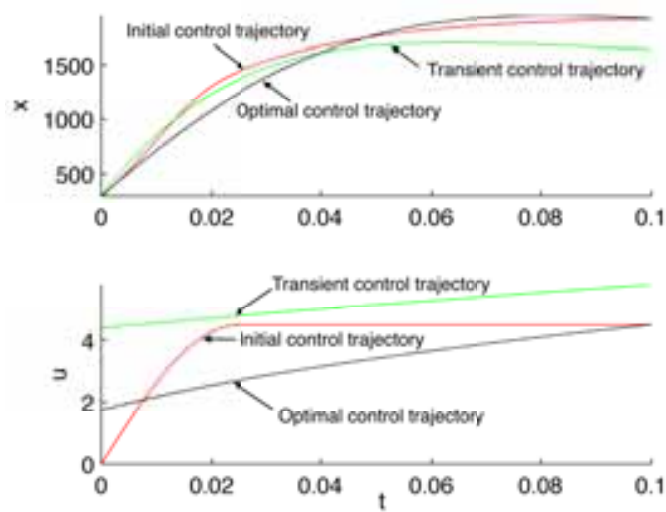

Figure 9: Variable functions w.r.t time (min time for 1900K) 

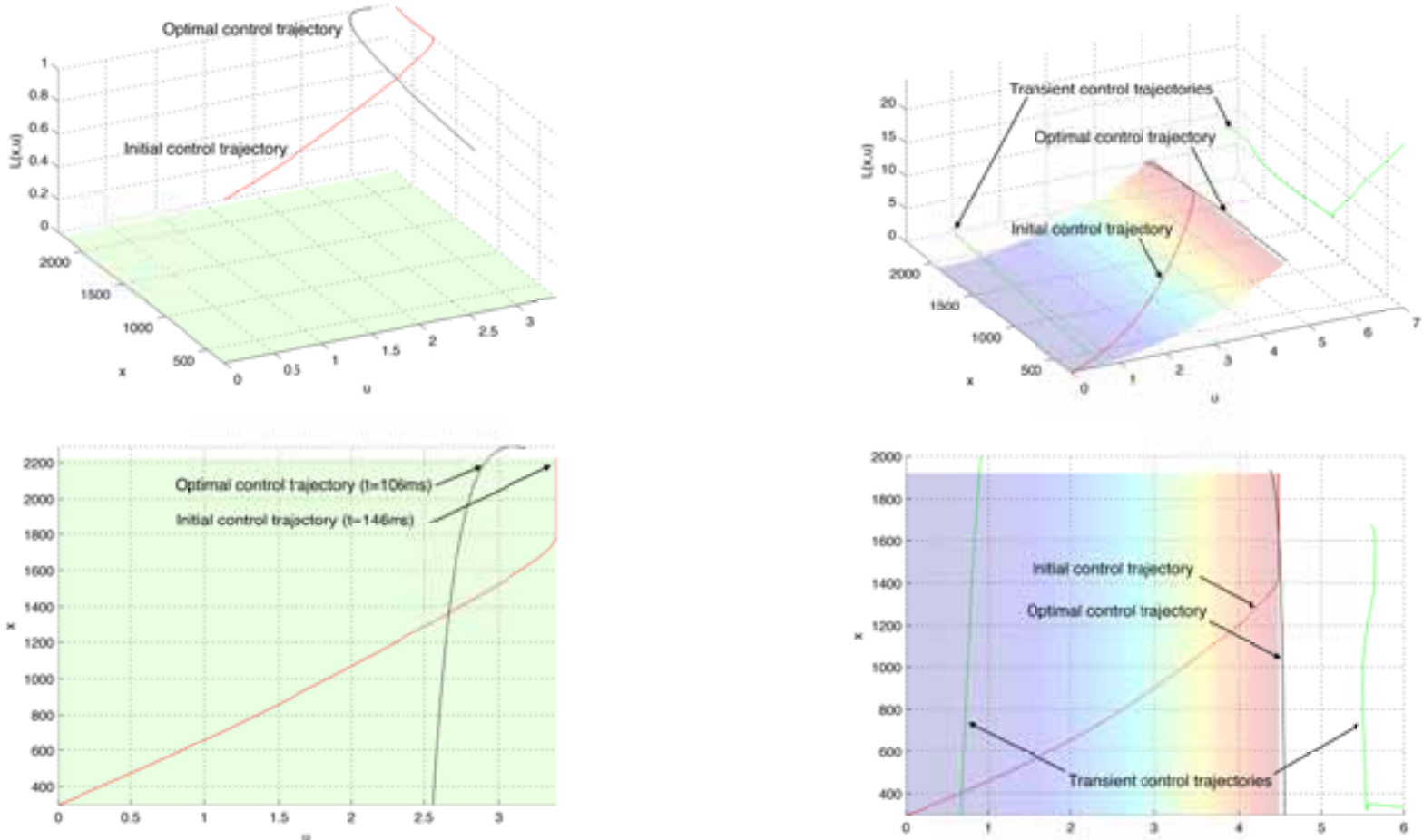

Figure 10: Control trajectory plot (min time for $2200 \mathrm{~K}$ )

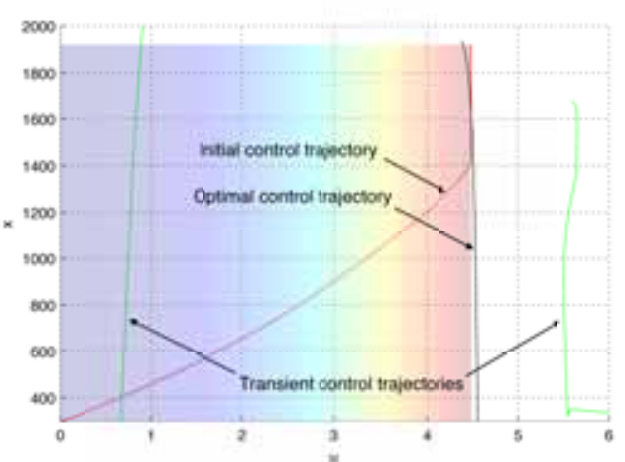

Figure 12: Control trajectory plot (min variance for 1900K)
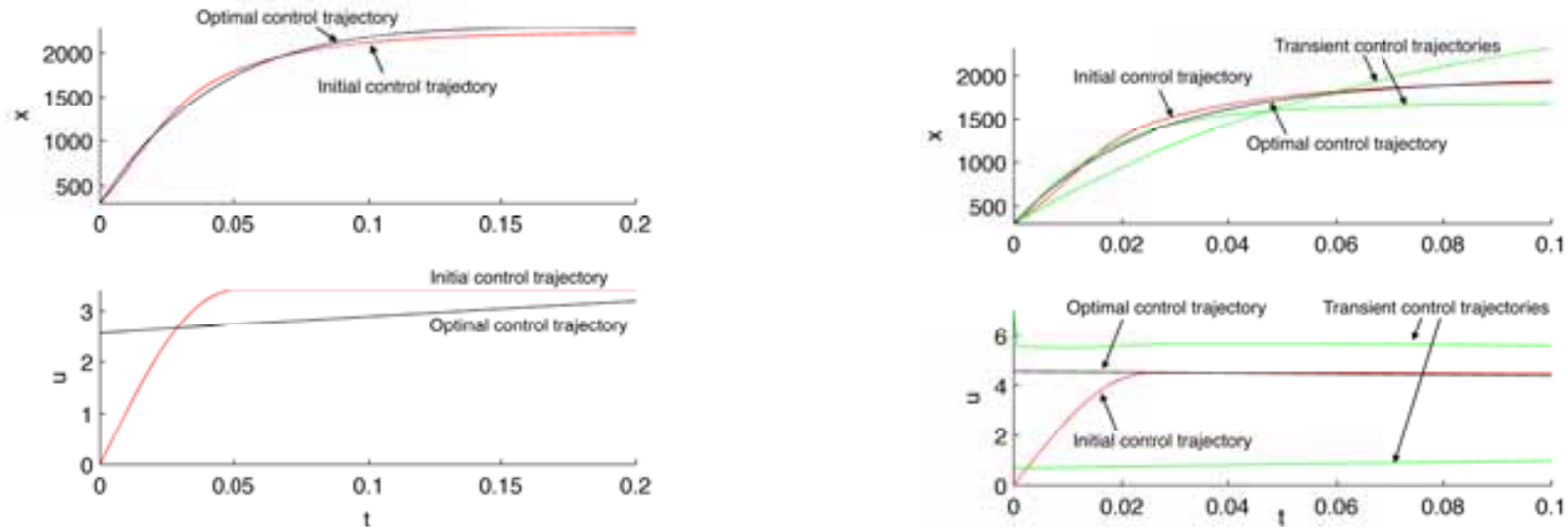

Figure 11: Variable functions w.r.t time (min time for 2200K)

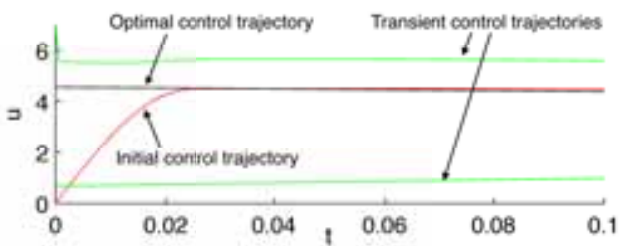

Figure 13: Variable functions w.r.t time (min variance for 1900K) 

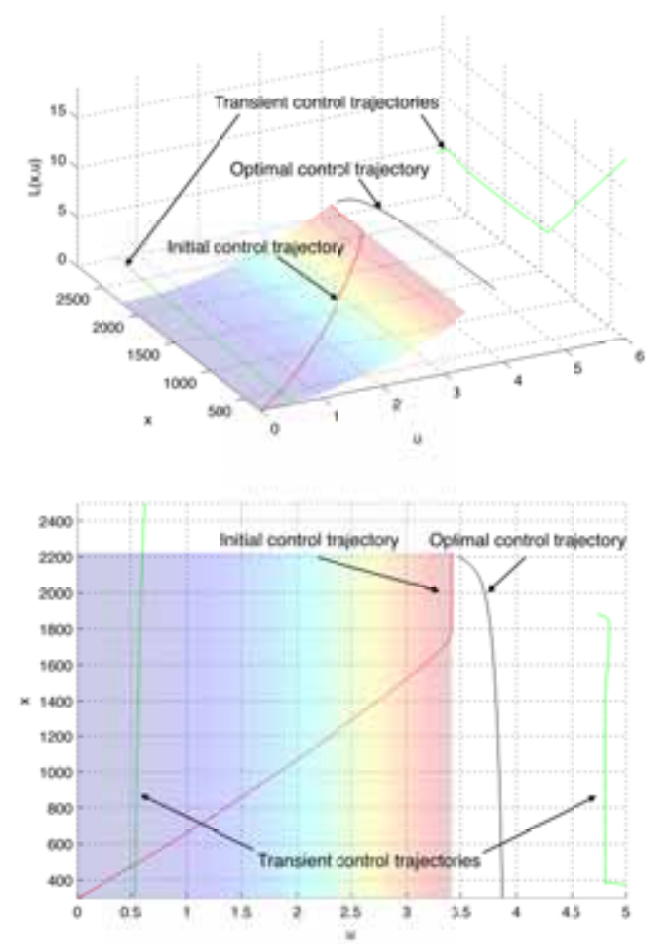

Figure 14: Control trajectory plot (min variance for 2200K)
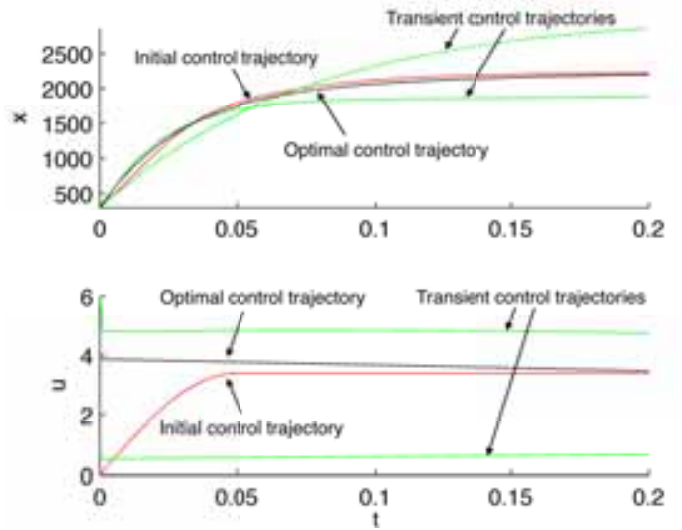

Figure 15: Variable functions w.r.t time (min variance for 2200K) 